\title{
ESTÁGIO DOCÊNCIA DURANTE A FORMAÇÃO DE MESTRANDOS NA ÁREA DE SAÚDE COLETIVA: UM RELATO DE EXPERIÊNCIA
}

\author{
TEACHING STAGE DURING TRAINING OF MASTERS IN THE COLLECTIVE \\ HEALTH AREA: A REPORT OF EXPERIENCE
}

\author{
Núbia Samara Caribé de Aragão, Sarah Santos Conceição, Cinthia Soares Lisboa, Carla \\ Alencar Cruz, Carlito Lopes Nascimento Sobrinho
}

Universidade Estadual de Feira de Santana - UEFS

\begin{abstract}
The objective of this article was to describe the experience of four master's students during the teaching internship, with a qualitative and descriptive approach. The internship is a curricular activity for undergraduate students stricto sensu, determined by the Coordination of Improvement of Higher Level Personnel, resolution 065/99, modified by resolution 013/00. The Postgraduate Program in Collective Health of the State University of Feira de Santana has the objective of training teachers and researchers in the area of Public Health. The teaching stage is offered in several disciplines of the health department of the university, linked to collective health, always under the supervision of a teacher. The internship was developed in the epidemiology and biostatistics disciplines of the undergraduate dental course, a six-hour semester workload, from March to July 2018. The experience allowed the first contact with teaching in higher education and the development of a work relevant in the execution of the disciplines in question, assisting teachers in the resolution of certain situations, in order to contribute to the process of student training. The teaching stage contributed in a significant way, as an enriching experience for the teacher training of future masters in collective health. It is considered that this study may support future reflections about teaching-learning, as well as the training of future higher education teachers.
\end{abstract}

Key words: Teaching; Public Health; Professional Training.

\section{Resumo}

O objetivo deste artigo foi descrever a experiência de quatro mestrandas durante $o$ estágio docência, com abordagem qualitativa $e$ descritiva. O estágio é uma atividade curricular para estudantes de cursos de pós-graduação stricto sensu, determinado pela Coordenação de Aperfeiçoamento de Pessoal de Nível Superior, resolução 065/99, alterada pela resolução 013/00. O Programa de Pós-Graduação em Saúde Coletiva da Universidade Estadual de Feira de Santana tem por objetivo a formação de profissionais docentes e pesquisadores na área da Saúde Coletiva. $O$ estágio docência é ofertado em várias disciplinas do departamento de saúde da universidade, vinculadas à saúde coletiva, sempre sob a supervisão de um professor. $O$ estágio foi desenvolvido nas disciplinas epidemiologia e bioestatística do curso de graduação em odontologia, carga horária semestral de sessenta horas, nos meses de março a julho de 2018. A experiência permitiu o primeiro contato com a docência no ensino superior e o desenvolvimento de um trabalho relevante na execução das disciplinas em questão, auxiliando os docentes na resolução de determinadas situações, a fim de contribuir no processo de formação dos alunos. $O$ estágio docência contribuiu de maneira significativa, como uma experiência enriquecedora para a formação docente das futuras mestres em saúde coletiva. Considera-se que este estudo poderá subsidiar reflexões futuras acerca do ensinoaprendizagem, bem como na formação dos futuros professores do ensino superior.

Palavras chave: Docência; Saúde Pública; Capacitação Profissional. 


\section{Introdução}

O Mestrado Acadêmico em Saúde Coletiva da Universidade Estadual de Feira de Santana, Bahia, foi instituído em 1997, passou a integrar o Programa de Pós-Graduação em Saúde Coletiva (PPGSC) no ano de 2004. O Curso de Mestrado do PPGSC tem como seus principais objetivos: qualificar docentes para o ensino de disciplinas no campo da saúde pública em cursos de graduação e pós-graduação; formar pesquisadores na área de saúde coletiva e contribuir para o desenvolvimento científico e tecnológico da área, com possibilidade de aplicação especial nos municípios da região do semiárido.

O propósito do programa de pós-graduação no nível de mestrado é de aprofundar o estudo do método científico na área da saúde coletiva, buscando qualificar os estudantes no estudo e aplicação do método científico para atender à expansão do ensino superior, além de contribuir para a formação de recursos humanos com elevado nível de qualidade para a prática do ensino $^{1,2}$.

$\mathrm{Na}$ pós-graduação, o estágio docência é uma oportunidade para praticar a indissociabilidade entre ensino, pesquisa e extensão, objetivando o domínio de conhecimentos, habilidades e competências que possibilitem desenvolver uma postura crítica sobre a prática docente ${ }^{3}$. É importante salientar que, o estágio docência é considerado um método que permite a primeira aproximação do mestrando com a prática profissional como docente, possibilitando o aprendizado relacionado ao saber fazer e saber julgar, o que implica em consequências consideradas importantes para compreensão das ações didáticas e pedagógicas existentes no cotidiano profissional $^{4}$.

Nesse sentido, a formação durante o estágio docência, pressupõe a reflexão da prática docente, por meio do envolvimento do mestrando em uma experiência concreta de ensino, supervisionada por um docente com experiência didático-pedagógica na área de saúde coletiva. Essa experiência visa oportunizar ao mestrando, o desenvolvimento de habilidades e competências que possam colaborar com a sua formação docente. Assim, as relações concebidas permitem trocas de conhecimentos, incentivando o professor a refletir sobre sua prática pedagógica e ao mestrando uma reflexão de como deve ser desenvolvida a sua prática docente $^{5}$.

O Estágio Docência foi estabelecido pela
Coordenação de Aperfeiçoamento de Pessoal de Nível Superior (CAPES) em 1999. Esse estágio é uma atividade curricular para estudantes de cursos de pós-graduação stricto sensu. Analisando a possibilidade de atuação na atividade docente. A CAPES na Resolução 065/99, alterada pela Resolução 013/00, tornou obrigatória para bolsistas de demanda social da CAPES a participação em estágio supervisionado ${ }^{2,6}$.

A normatização da atividade de estágio docência está contida na Portaria no 76, de 14 de abril de 2010, que revoga a Portaria no 52, de 26 setembro de 2002 da CAPES, estabelecendo que o mestrando/doutorando sejam inseridos em atividades de ensino sob a supervisão de um professor $^{6}$. De acordo com essa portaria, fica estabelecido que a duração mínima do estágio docência é de um semestre para o mestrado e de dois semestres para o doutorado, sendo as atividades do estágio docência compatíveis com a área de conhecimento do programa de pósgraduação realizado pelo pós-graduando ${ }^{6}$.

De acordo com as orientações da CAPES, o Programa de Pós-Graduação em Saúde Coletiva da Universidade Estadual de Feira de Santana (PPGSC/UEFS), tem por objetivo a formação de profissionais docentes e pesquisadores na área da Saúde Coletiva. Para essa formação, a UEFS oferta o estágio docência, com carga horária mínima de 30 horas, em vários componentes curriculares da área de saúde coletiva, pertencentes aos diversos cursos de graduação do Departamento de Saúde (DSAU), sempre sob a supervisão de um professor.

Considerando a importância que o estágio docência apresenta para a formação do estudante de pós-graduação, o presente estudo tem como objetivo descrever a experiência vivenciada por mestrandos em Saúde Coletiva do PPGSC/UEFS durante o estágio docência, no componente curricular Bioestatística e Epidemiologia, com discentes de graduação em Odontologia, no primeiro semestre de 2018.

\section{Metodologia}

Trata-se de um relato de experiência, com abordagem qualitativa e descritiva, sobre o estágio docência de quatro mestrandas do Programa de Pós-graduação em Saúde Coletiva da Universidade de Feira de Santana (UEFS), com diferentes formações durante a graduação: duas enfermeiras, uma cirurgiã-dentista e uma nutricionista, acerca da experiência vivenciada durante o estágio docência no componente curricular Bioestatística e Epidemiologia, do 
Curso de Graduação em Odontologia da UEFS, com carga horária semestral de sessenta (60) horas, no primeiro semestre do ano de 2018.

A experiência aconteceu no período de março a julho de 2018, semanalmente, toda quinta-feira. A participação das mestrandas nas disciplinas ocorreu sob a supervisão de dois professores, cada um responsável por uma turma, com aproximadamente 20 estudantes. Embora seja preconizado 30 horas para o estágio docência, as mestrandas cumpriram a carga horária de 60 horas.

O componente curricular Bioestatística e Epidemiologia é de caráter teórico-prático, sendo obrigatória para a graduação, tendo como objetivos: reconhecer os fundamentos científicos em que se baseia a Epidemiologia para o estudo do processo saúde-doença em populações humanas; utilizar técnicas de pesquisa epidemiológica; calcular e analisar criticamente, os principais indicadores de saúde na população; distinguir os diferentes tipos de estudos epidemiológicos, suas principais aplicações, bem como, as vantagens e desvantagens de cada tipo de estudo; reconhecer os usos e finalidades da Bioestatística no campo da pesquisa e na prática da saúde coletiva; apresentação dos Sistemas de Informação em Saúde e fontes de dados discutindo sua utilização em Epidemiologia.

Inicialmente, foi realizada reunião entre os docentes e mestrandas a fim de realizar a leitura do plano de ensino do componente curricular para conhecer os objetivos, conteúdos, metodologias, avaliações previstas e referências bibliográficas a serem utilizadas. A partir daí, foram organizadas as aulas que cada mestranda iria ministrar de acordo com o cronograma para atingir os objetivos propostos. Na sequência, no primeiro dia de aula houve o contato com a turma, realizando-se a apresentação dos professores e mestrandas e, logo após a apresentação dos estudantes e suas expectativas em relação ao componente curricular.

As mestrandas atuaram na preparação de planos de aula e do material didático, elaboração e exposição de aulas teóricas e práticas, aplicação e correção de exercícios propostos em sala, preparação e correção das avaliações. Durante todas as etapas, a execução das atividades acontecia, após apreciação e aprovação pelos professores. Os resultados descritos neste relato de experiência foram obtidos durante a realização do estágio docência e sistematizados no relatório do estágio elaborado pelos mestrandos sob a supervisão dos professores e descreve a participação e envolvimento das mestrandas durante o processo de convivência com os professores e discentes do componente curricular.

Ao final do estágio docência foi elaborado um relatório descrevendo as atividades realizadas durante o estágio, a avaliação do componente curricular, as percepções da experiência vivenciada em sala de aula, as facilidades e limitações enfrentadas durante o estágio, as estratégias criadas para o melhor enfrentamento das limitações descritas, assim como a contribuição do estágio para a formação didático-pedagógica dos mestrandos.

\section{Resultados e Discussão}

Das 10 aulas teóricas descritas no cronograma de disciplina, as mestrandas elaboraram e apresentaram cinco, versando sobre os seguintes temas: Indicadores de Morbidade e Mortalidade, Representação Gráfica e Tabular (teórica e prática em laboratório de informática) e duas sobre Estudos Epidemiológicos (Intervenção, Coorte, Casocontrole e Transversal, Ecológico, Descritivo). Além de elaborarem as avaliações incluindo os conteúdos apresentados em sala de aula, durante as duas unidades e aplicadas em datas previamente agendadas.

Foram utilizados recursos complementares para melhorar a apresentação e aprendizagem do conteúdo, com o auxílio das referências bibliográficas de acordo com os temas abordados. As aulas teóricas foram ministradas com o uso de recursos audiovisuais (Datashow) e quadro, sendo complementados com discussões de exemplos reais do cotidiano, trabalhos em grupo com oficina de artigos, resoluções de questões em sala, lista de exercícios para casa e aulas práticas em laboratório de informática.

A experiência com o estágio docência permitiu às mestrandas o primeiro contato com a docência no ensino superior. Tendo em vista que, - estágio docência é caracterizado por acompanhar o professor durante o desenvolvimento do componente curricular, desde o seu planejamento até a execução das atividades. É importante destacar que, esse estágio é marcado por desenvolver a prática docente, com a finalidade de promover a reflexão crítica em torno da atividade pedagógica. O docente constitui sua identidade e representa seus papeis não só com seus pares, mas também na interação do cotidiano com seus alunos, já que tal representação não se dá de maneira isolada, mas sempre na relação com o outro ${ }^{7,8}$.

Como um campo disciplinar e investigativo, a didática, ainda é bastante questionada no 
campo da educação, mas, também, apresenta-se em expansão. Quanto aos questionamentos, são conhecidas e identificáveis as resistências em reconhecê-la em seu status científico e em seu papel na formação de professores ${ }^{9}$. Por outro lado, é forçoso admitir que em boa parte dos cursos de formação de professores persiste a didática prescritiva e instrumental, acompanhada por conteúdo e métodos tradicionais ${ }^{10}$, num contexto em que as questões de ensinoaprendizagem se cercam de complexidade cada vez maior, exigindo revisões, atualizações, relações com outros campos de investigação.

Durante o estágio docência as quatro mestrandas puderam compartilhar saberes e práticas de suas experiências profissionais e acadêmicas com os discentes e com os professores responsáveis pelo componente curricular, proporcionando às pós-graduandas o desenvolvimento de práticas didáticas junto às atividades de ensino, a vivência da relação professor-aluno no contexto da sala de aula e a compreensão da importância da docência na formação profissional.

Diante da temática proposta, antes de cada aula lecionada, às mestrandas construíam um plano de aula, que segundo Libâneo ${ }^{11}$ é um instrumento que sistematiza todos os conhecimentos, atividades e procedimentos que se pretende realizar numa determinada aula, tendo em vista o que se espera alcançar como objetivos junto aos alunos. Nessa perspectiva, o contato com plano de aula é parte fundamental para nortear e conduzir a prática docente.

No transcorrer da disciplina, houve a oportunidade de participar e contribuir nas aulas práticas, em que os graduandos puderam empregar os conhecimentos abordados nas exposições teóricas, utilizando o programa do Departamento de Informática do Sus (DATASUS) para a realização de um diagnóstico situacional de saúde de uma determinada população e município. Foi um momento marcado por descobertas interessantes, em que os discentes compreenderam a importância dos enfoques dados aos sistemas de informação em saúde e a importância da qualidade dos dados secundários para a construção de diagnósticos de saúde no âmbito da saúde pública.

Portanto, percebeu-se a necessidade destas atividades para que os discentes se apropriem dos conhecimentos do referido componente curricular. Corroborando as perspectivas de Tardif $^{12}$, quando afirma que o espaço educativo extrapola as informações sobre os conteúdos, devendo ser reflexivo e com debates capazes de auxiliar na compreensão de aspectos político- econômicos que estruturam a sociedade.

Outra etapa extremamente importante para esta experiência do estágio docência foi o processo avaliativo realizado na disciplina. As mestrandas tiveram a oportunidade de elaborar questões de prova, aplicar atividades avaliativas e posteriormente corrigi-las. Este processo foi visto como um grande desafio, uma vez que foi possível problematizar sobre a complexidade do processo avaliativo de alunos, conclui-se que a avaliação como algo processual e, portanto, que transcende a avaliação pontual representada pela realização de provas de avaliação de desempenho.

É preciso salientar que, as relações construídas em sala de aula, os diálogos, o comportamento dos discentes, são fatores inerentes ao processo avaliativo. Esta percepção confirma as afirmações de Luckesi $^{13}$, ao sinalizar que o ato de avaliar implica buscar soluções para os problemas encontrados. Dessa forma, a avaliação dos graduandos do curso de odontologia foi realizada levando em consideração a participação durante as aulas, execução dos exercícios propostos, relação com os professores, além de duas avaliações escritas no decorrer da disciplina.

Por meio do planejamento, elaboração e realização de aulas foi possível experimentar o "Ser Docente", buscando conhecimentos metodológicos, atualização científica objetivando promover da melhor forma possível à compreensão dos discentes com relação aos conteúdos apresentados e contribuir com o processo de ensino-aprendizagem. Neste sentido Freire $^{14}$, afirma que ensinar não é transferir conhecimento, mas criar possibilidades para a sua produção ou a sua construção.

Vale destacar que, nos cursos de pósgraduação stricto senso a concretização dos saberes da prática educativa geralmente não se constitui um elemento primordial, pois a pesquisa e o aprofundamento dos saberes específicos do campo da saúde são priorizados. Nessa perspectiva, é essencial que o saber pedagógico da docência universitária seja reconhecido por sua grande relevância. Este é o grande desafio que se impõe a estes programas, romper com o modelo que prestigia basicamente a competência em pesquisa no seu campo específico de formação, e acrescentar as preocupações com a dimensão ensinoaprendizagem de forma mais coerente ${ }^{15}$.

\section{Conclusões}

O processo de aprender-ensinar vivenciado 
faz parte de um momento importante na formação do mestre em saúde coletiva. É imprescindível a aproximação dos estudantes de pós-graduação em nível mestrado com o campo de prática profissional. A atividade do estágio docência é um espaço amplo para discussões teóricas, metodológicas e práticas.

A experiência com a prática do estágio docência proporcionou o aprendizado a respeito de estratégias metodológicas do ensino e aprendizagem, além da compreensão que o professor é um mediador e deve entender sobre o tema que é ministrado, mas, sobretudo, ter capacidade para gerenciar conflitos e estabelecer estratégias tendo em vista, a garantia de uma aprendizagem significativa, propiciando descobertas e oportunizando a realização do processo ensino-aprendizagem.

Verificou-se que 0 estágio docência proporcionou às mestrandas um momento enriquecedor desde a primeira reunião, até os momentos experienciados com os alunos em sala de aula. É importante evidenciar que o professor orientador é marcante durante todo este processo, o aprendizado só acontece com a prática, observação da experiência e descobertas a cada nova aula.

O estágio docência contribuiu de maneira significativa, como uma experiência enriquecedora para a formação docente das futuras mestres em saúde coletiva. Nesse sentido, considera-se que este estudo poderá subsidiar reflexões futuras acerca do ensino-aprendizagem, bem como na formação dos futuros professores do ensino superior.

\section{Referências}

1. Bastos CCBC. Docência, pós-graduação e a melhoria do ensino na universidade: uma relação necessária. Educere et Educare, 2007; 2 (4): 103-112.

2. Joaquim $\mathrm{N}$ de $\mathrm{F}$, Boas $\mathrm{AAV}$, Carrieri $\mathrm{A}$ de P. Estágio docente: formação profissional, preparação para o ensino ou docência em caráter precário? Educaçao e Pesquisa: Revista da Faculdade de Educação da Universidade de São Paulo, 2013; 39(2):351-65.

3. Lourenço CD da S, Lima MC, NArciso ERP. Formação pedagógica no ensino superior: o que diz a legislação e a literatura em Educação e Administração? Rev da Avaliação da Educ Super. 2016;21(3):691-717, nov.

4. Freire AM. Concepções orientadoras do processo de aprendizagem do ensino nos estágios pedagógicos. Lisboa, 2001, 25fls. . Disponível http://www.educ.fc.ul.pt/recentes/mpfip/pdfs/a freire.pdf>. Acesso em: 30 jul. 2018.

5. Nunes RV, Fraga AB."Alinhamento astral": o estágio docente na formação do licenciado em Educação Física na ESEF/UFRGS. Pensar a Prática. 2006;9(2):297312.

6. Oliveira ML, Silva NC. Estágio de Docência na Formação do Mestre em Enfermagem: Relato de Experiência. Enferm em Foco. 2012;3(3):131-4.

7. Berger PL, Luckmann T. A construção social da realidade: tratado de sociologia do conhecimento. 16. ed. Petrópolis: Vozes, 1998.

8. Goffman E. A representação do eu na vida cotidiana. 17. ed. Rio de Janeiro, Petrópolis, Vozes, 2011.

9. Libâneo JC. Didática e docência: formação e trabalho de professores da educação básica. In: CRUZ, Gisele Barreto et al (org.). Ensino de didática: entre recorrentes e urgentes questões. Rio de Janeiro: Ed. Quartet, 2014.

10. Libâneo JC. O ensino da Didática, das metodologias específicas e dos conteúdos específicos do ensino fundamental nos currículos dos cursos de Pedagogia. Revista Brasileira de Estudos Pedagógicos, Brasília, 2010; 91(229): 562-583.

11. Libâneo JC. Organização e gestão escolar: teoria e prática. Goiânia: Alternativa, 1993.

12. Tardif M. Os professores diante do saber: esboço de uma problemática do saber docente. In: Tardif M. Saberes docentes e formação profissional. 12. ed. Petrópolis, RJ: Vozes, 2002, p. 31-55.

13. Luckesi CC. Avaliação da Aprendizagem: compreensão e prática. Jornal do Brasil, $27 \mathrm{abr}$ 2000.

14. Freire P. Pedagogia da autonomia: saberes necessários à prática educativa. São Paulo: Paz e Terra; 1996.

15. Santos LP de S, Miranda SS, Graça CC, Sobrinho CLN. Estágio Docência Na Formação Do Mestre Em Saúde Coletiva: Relato De Experiência Stage Teaching in Formation of the Master in Public Health: Experience Report. 2015;418-24.

\section{Endereço para Correspondência}

Universidade Estadual de Feira de Santana - UEFS

Av. Transnordestina, s/n, Novo Horizonte - BA

CEP.: 44036-900 
e-mail: nscaribe@hotmail.com

Recebido em 20/09/2018

Aprovado em 06/06/2019

Publicado em 13/02/2020 